\title{
TRANSPARENCIA Y DIFUSIÓN DE LAS TECNOLOGÍAS DE LA INFORMACIÓN EN MUNICIPIOS ARGENTINOS
}

\author{
María Verónica Alderete \\ Instituto de Investigaciones Económicas y Sociales del Sur, CONICET, \\ Argentina \\ mvalderete@iiess-conicet.gob.ar
}

\section{RESUMEN}

Este trabajo estudia el principio de transparencia como uno de los ejes del paradigma de gobierno abierto. El principal objetivo es examinar si existe algún agrupamiento de los municipios según las variables transparencia y difusión de las Tecnologías de Información y Comunicación (TIC). En consecuencia, se busca clasificar a los municipios capitales de provincia en Argentina en función a tales indicadores. Con este fin, se utiliza el Índice de Transparencia Municipal de Argentina (ITMA) que analiza la transparencia activa presupuestaria y transparencia pasiva; así como indicadores de acceso y uso de TIC del Instituto Nacional de Estadísticas y Censos (INDEC). Mediante un análisis de conglomerados de $\mathrm{k}$ medias, se distinguen dos grupos de municipios: municipios de alta transparencia y difusión de las TIC; y municipios de baja transparencia y difusión de las TIC. Tales conglomerados se diferencian en relación al tamaño de los municipios y al ingreso o recursos económicos.

Palabras clave: Transparencia, Tecnologías de la Información, Municipios, Argentina. 


\title{
TRANSPARENCY AND DISSEMINATION OF INFORMATION TECHNOLOGIES IN ARGENTINE MUNICIPAL GOVERNMENTS
}

\begin{abstract}
This study examines the principle of transparency as one of the pillars of the paradigm of open government. Its main objective is to determine whether there is any grouping of municipal governments according to transparency and the dissemination of information and communications technologies (ICTs). Consequently, it seeks to classify the municipal governments of Argentina's provincial capitals based on these indicators. For this purpose, it uses the Argentine Municipal Transparency Index (ITMA), which measures active budget transparency and passive transparency, and indicators of ICT access and use from the National Institute of Statistics and Censuses (INDEC). Through k-means cluster analysis, it distinguishes two groups of municipal governments: those with high transparency and ICT dissemination and those with low transparency and ICT dissemination. These clusters differ in the size of the municipalities and their income or economic resources.
\end{abstract}

Keywords: Transparency, Information technologies, Municipal governments, Argentina. 


\section{INTRODUCCIÓN}

La transparencia y el acceso a la información gubernamental se convirtieron en todo el mundo en los factores claves para lograr la confianza en los gobiernos, permitir la participación democrática y la toma de decisiones racionales, y prevenir la corrupción, entre otras funciones. Tanto la apertura de datos como la transparencia son factores esenciales para asegurar la confianza de los ciudadanos en los gobiernos y en la democracia en su conjunto (Cullier y Piotrowski 2009, Kolstad y Wiig 2009, Kim et al. 2005).

El surgimiento de un nuevo paradigma de gobierno abierto se basa en los principios de transparencia, compromiso y participación ciudadana, y tecnología e innovación. En este marco se crea la Alianza para el Gobierno Abierto, una organización multilateral cuyo objetivo consiste en fomentar la cultura de gobierno abierto, generar empoderamiento a los ciudadanos, brindar resultados y promover los ideales de la apertura y la participación del siglo XXI. En este sentido, aquellas iniciativas que buscan perfeccionar los mecanismos de transparencia de la gestión de gobierno son consideradas buenas prácticas.

Una política de los gobiernos en torno a la modernización de la administración pública ha sido el desarrollo del gobierno electrónico, el cual consiste en modernizar el canal de comunicación y de interacción entre los gobiernos y los ciudadanos. Alrededor del mundo, los gobiernos han adoptado el gobierno electrónico tanto para obtener beneficios a partir de la provisión de los servicios públicos como beneficiar a la ciudadanía que usualmente utiliza tales servicios (Weeradoky et al. 2016, Gupta et al. 2016, Chen 2010, Teo et al. 2008, Jaeger 2003). Asimismo, se entiende al gobierno electrónico como la relación de los ciudadanos con el gobierno a través de la utilización de los servicios y datos en línea (Curtin, Sommer, Sommer 2003).

En este contexto, surgió una serie de iniciativas con el fin de promover prácticas de gobierno que relacionan las nuevas tecnologías con la Administración pública y el Gobierno Abierto (Mariñez 2017, Naser y Ramírez Alujas 2017, CLAD 2016, Criado 2016, Ramírez-Alujas y Güemes 2013). Por su parte, esta noción de acercar las administraciones públicas a los ciudadanos es fundamental para quienes consideran necesaria una mayor transferencia de competencias y responsabilidades del gobierno central hacia los niveles territoriales o municipales de gobierno (Bonivento 2016). Este proceso tuvo lugar en América Latina durante los años ochenta y noventa del siglo pasado, bajo la idea de búsqueda de proximidad y de 
reforma de descentralización. En la medida en que se suministren mejores y mayores mecanismos de interacción social entre los ciudadanos y los municipios, mayores serían las ventajas de la proximidad administrativa y la acción colaborativa (Hernández Bonivento 2016).

Sin embargo, en el nivel local, los estudios comparativos desde el punto de vista de la transparencia son aún escasos. Ciucci et al. (2019) realizan una revisión de los índices de transparencia en América Latina. Un estudio influyente en la región ha sido el desarrollado por Hernández Bonivento (2016) para los municipios de Chile. A través de la construcción de datos originales y el uso de fuentes secundarias, Hernández Bonivento elaboró el Índice Institucional para el Gobierno Abierto Municipal, el cual mide las dimensiones de transparencia (activa y pasiva), participación ciudadana, y colaboración en 345 municipios de Chile.

En Argentina, la sanción de la Ley de Acceso a la Información Pública el 15 de septiembre de 2016 da inicio al proceso de transparencia en la administración pública. El objetivo de la misma es obligar a los tres poderes del Estado y a los organismos relacionados que sean financiados por el estado (empresas públicas, universidades, entre otros) tanto a difundir información púbica mediante datos abiertos (transparencia activa) como a responder las solicitudes de información que eleve cualquier ciudadano en un plazo no mayor a un mes (transparencia pasiva). Las provincias de Argentina presentan una gran divergencia con respecto a las normas que regulan el derecho de acceso a la información y a los criterios para poner a disposición de los ciudadanos la transparencia de los actos de gobierno. Por un lado provincias donde no existe una ley para ejercer el derecho, otras que cuentan con una legislación pero con muchas deficiencias (Farioli 2015).

El análisis a nivel municipal del principio de transparencia en un país federal como Argentina plantea ciertas dificultades. Por un lado, las leyes orgánicas de los municipios de cada provincia difieren y determinan la obligatoriedad de publicación de ciertos datos por parte de los municipios. Por otro lado, aun cuando los municipios cumplieran lo establecido por la ley, esta suele determinar la obligatoriedad de un piso mínimo, por lo cual los municipios pueden ejercer una política de transparencia mucho más proactiva que la legal.

A nivel de los municipios, la ciudadanía logra acercarse a los ámbitos de gobierno y, por tanto, se evidencia de forma más clara un cambio en el rol del ciudadano (Montoya Morales y Zhardi 2016). Mediante las tecnologías de la información y comunicación (TIC), la ciudadanía asume un papel 
más activo en la toma de decisiones políticas (Aström et al. 2012). Sin embargo, si el acceso a las nuevas tecnologías no es universal, para toda la población, surge el riesgo de generar una brecha digital entre quienes pueden utilizar los servicios de gobierno electrónicamente y aquellos que no (Banco Mundial 2016, Alderete 2018).

Existen diversas teorías que brindan un aporte para explicar las causas que originan el proceso de digitalización, modernización y adopción de las nuevas tecnologías en las administraciones públicas. Por un lado, se encuentran la teoría de difusión de la innovación (Rogers, 1995) y el modelo de aceptación de la tecnología (Davis, 1989). De igual manera, el concepto de ciudades inteligentes o smart cities introduce como una de sus dimensiones fundamentales el smart governance (Albino, Beraldi y Dangelico 2015, Anthopoulos y Fitsilis 2010).

En este estudio, se propone identificar grupos de municipios con base en sus niveles de transparencia y de difusión de las TIC, así como determinar si existen diferencias significativas entre estos grupos en lo relativo con su tamaño y recursos económicos. Se plantea el siguiente interrogante: ¿son los municipios con una mayor penetración de las TIC, los municipios con mayor grado de transparencia? Con este fin, se realiza un análisis de conglomerado para clasificar a los municipios en función a estas variables. Por último, se examina si existen diferencias entre los conglomerados encontrados respecto a ciertas variables de interés. El objetivo no consiste en proveer una explicación completa de los niveles de transparencia en municipios de Argentina, sino en ofrecer una taxonomía de los municipios capitales de provincia de Argentina sobre la base del único índice de transparencia a la fecha en el país el, Índice de Transparencia Municipal de Argentina (ITMA).

El trabajo se estructura de la siguiente manera. En primer lugar, se describe el marco teórico que define los conceptos teóricos involucrados e incluye una revisión de la bibliografía empírica. En segundo lugar, se esboza el estado del arte en torno a los índices de transparencia existentes en Argentina y estadísticas descriptivas sobre TIC a nivel regional. En tercer lugar, se plantea la metodología y la fuente de datos empleada. En cuarto lugar, se presentan los resultados hallados. Por último, se comparte alguna discusión y consideraciones finales. 


\section{MARCO TEÓRICO}

\section{CONCEPTO DE GOBIERNO ABIERTO, TRANSPARENCIA}

El paradigma de gobierno abierto u open government data sostiene que los datos de carácter público sean de acceso abierto y estén disponibles libremente para cualquiera. De esta manera, el gobierno abierto puede generar servicios gubernamentales más eficientes y accesibles entre todos los stakeholders o tomadores de decisiones, más transparencia y un empoderamiento de los ciudadanos a través del acceso a la información y a una administración más eficiente (Oszlak y Kaufman 2014, Herrero y Gentili 2015). A su vez, genera un cambio estructural en la tendencia que hasta el momento se observaba en la evolución del denominado gobierno electrónico o gobierno digital extendiendo sus beneficios más allá de la simplificación de trámites y de la mayor transparencia de información por parte de los gobiernos de la gestión (Concha y Naser 2012). Este cambio implica una colaboración interinstitucional (Mariñez 2017, Criado 2016, Ramírez-Alujas y Güemes 2013); es decir, un fuerte componente relacional entre los funcionarios públicos, los ciudadanos y el sector privado en la cocreación de tales innovaciones.

El proceso de transparencia no solo implica la transparencia activa, entendida como el nivel en que el municipio publica de oficio información pública de sus actividades, sino también la transparencia pasiva, es decir, el grado en que el municipio da respuesta a las solicitudes de información pedidas por los ciudadanos o las organizaciones de la sociedad civil en tiempo y forma. Por otro lado, "la transparencia puede verse desde dos dimensiones: la activa (que vuelve operativo el deber del Estado de proveer información) y la pasiva (que vuelve operativo el derecho de acceso a la información) (Alderete y Linares 2017)”. A su vez, se puede definir a la transparencia activa como la obligación de la administración pública de publicar y mantener actualizados ciertos ítems, datos o documentos que contienen información pública relevante. Se presume que los datos considerados relevantes producidos por la Administración deberían ser visibles, localizables, accesibles y expresados con claridad. Si bien tradicionalmente se utilizaban archivos documentales y repositorios, la tendencia moderna es la de suministrar la información online, a través de la web.

Asimismo, la transparencia pasiva se puede caracterizar como un derecho de la ciudadanía a recibir la información pública que solicite. Para cumplir este objetivo, son necesarios canales adecuados que envíen la solicitud, y un tratamiento y entrega de información particular para cada caso. El objetivo 
es que dicho procedimiento sea amigable, ágil y eficiente. Al igual que la transparencia activa, la orientación moderna es que el procesamiento de la solicitud, su gestión y respuesta sea a online, mediante la web, y que cualquiera pueda observar el estado de la solicitud y acceder a la respuesta.

\section{RELACIÓN ENTRE GOBIERNO ABIERTO Y TIC}

La revolución informática es la manera en que la información tecnológica es utilizada para mejorar las transacciones entre los gobiernos y otros actores de la sociedad y aparece como un medio para mejorar la gobernanza y el proceso democrático (Brewer, Neubauer y Geiselhart 2006). Las mejoras en las transacciones entre el gobierno o las organizaciones gubernamentales y los ciudadanos son propiciadas por las TIC que brindan flexibilidad en el tiempo sin necesidad de trasladarse (García-Sánchez, RodríguezDomínguez, y Frías Aceituno 2013, Ke y Wei 2004). Mariñez (2017) señala que el uso de las tecnologías de información y comunicación (web 2.0) permite un trabajo colaborativo entre ciudadanos, funcionarios públicos y sector privado, con el fin de co-crear métodos, técnicas y habilidades.

La creciente popularidad del gobierno electrónico lo convierte en un elemento importante para el desarrollo de la sociedad del conocimiento. Se entiende por gobierno electrónico a los procesos de simplificación y ejecución de la información, comunicación y de intercambio dentro y entre las instituciones gubernamentales, así como entre las instituciones gubernamentales y los ciudadanos u organizaciones (Meijer 2012). Sin embargo, el uso de las TIC en las relaciones del gobierno, conocido como gobierno electrónico, no necesariamente implica gobierno abierto. La Alianza Internacional para el Gobierno Abierto (AGA), al respecto sostiene que no todas las reformas de e-gobierno favorecen la apertura, para ser considerados compromisos relacionados a los valores de gobierno abierto. Para que esto ocurra es necesario articular la tecnología con aspectos claros de acceso a la información pública, participación ciudadana o rendición de cuenta. En este sentido, las TIC pueden facilitar la transparencia y participación, pero no tienen la capacidad por sí mismas de generar mayor democracia y compromiso ciudadano, uno de los terceros pilares del gobierno abierto (Cruz et al. 2012). Al respecto, Naser y Ramírez Alujas (2017) agregan que la transparencia y el uso de las TIC no son suficientes para alcanzar una mayor apertura de gobierno, sino que es necesario desarrollar una reforma de la administración pública que sea adecuada para responder a las exigencias de una democracia participativa y colaborativa.

Durante los últimos años, la difusión de las TIC ha permitido que las actividades del sector público así como los mecanismos mediante los cuales 
se canaliza la información del sector se desarrollen fácilmente, dando origen a la transparencia (Cerrillo i Martínez 2012, Fenster 2010, Fung et al. 2007, Ackerman 2006, Cerrillo i Martínez 2005). Actualmente, existe amplio consenso en la literatura de que la transparencia se canaliza fundamentalmente a través de los medios electrónicos (Meijer 2009, Grimmelikhuijsen 2009, Curtin y Meijer 2006, Cerrillo i Martínez 2005).

Los funcionarios públicos y tomadores de decisiones pueden beneficiarse del conocimiento que está difundido en la sociedad para desempeñarse mejor y generar mejores vínculos con la sociedad (Noveck 2009). Por ese motivo, las TIC poseen un efecto en la relación entre los gobiernos y los ciudadanos en la medida en que pueden ampliar y diversificar los canales de la administración pública y abrir nuevos caminos de comunicación para generar, distribuir y usar los datos y la información pública que sea relevantes para los ciudadanos (Alderete 2018).

Las TIC tendrían poco valor social si los ciudadanos no pudieran utilizar los servicios o interactuar en los procesos políticos de distintas maneras (Helbig et al. 2009). Las nuevas tecnologías han ampliado enormemente el elenco de alternativas o formas de articular en la práctica el derecho a participar en la democracia (Ebbers, Jansen y van Deursen 2016, Lau et al. 2008, Grant y Chau 2005, Grönlund y Horan 2004, Ke y Wei 2004). La participación electrónica puede contribuir al desarrollo de nuevas formas de colaboración entre el gobierno y las personas y refuerza las necesidades de la gente (Naciones Unidas 2016). En este sentido, los temas vinculados a la brecha digital (van Deursen y Van Dijk 2011, Dewan's y Riggins 2005), tanto en términos de acceso (entre quienes poseen o no la tecnología) como de uso de las TIC (entre quienes usan o tienen habilidad para usar la tecnología), afectan el desarrollo del gobierno electrónico mediante una serie de canales: impactando en la demanda de los servicios de gobierno electrónico, restringiendo la utilidad de algunas aplicaciones o determinando las preferencias sociales por las TIC en ciertos dominios públicos. Sin embargo, algunos autores (Villoria y Ramírez Alujas 2013) explican para América Latina, mediante el uso de la teoría política, el fracaso de las TIC en el gobierno respecto al desarrollo de una nueva época que permitirá a las administraciones ser más abiertas y eficaces.

\section{RELACIÓN TRANSPARENCIA Y OTRAS VARIABLES}

Ciertos factores propios de los municipios brasileros tienen incidencia sobre los niveles alcanzados de transparencia a nivel municipal (Cruz et al. 2012). En primer lugar, el autor encuentra que los ingresos presupuestarios posee un efecto significativo y positivo sobre el nivel de transparencia 
municipal. Asimismo, Camisón-Zornoza et al. (2004) encuentra una correlación positiva y significativa entre el nivel de gobierno electrónico y el tamaño de los municipios. Este resultado explica que la implementación de las iniciativas en materia de transparencia requiere de ingresos y recursos económicos. A nivel internacional, la evidencia empírica encuentra que el gobierno electrónico demanda inversiones en TIC que los países más pobres no pueden solventar. Ciertos autores han analizado la relación entre PBI y gobierno abierto a nivel países (Alderete 2018, Khalil 2011, Siau y Long 2009, Singh et al. 2007). Entre ellos, Khalil (2011) halla que el PBI posee una correlación positiva con el desarrollo del gobierno electrónico. $\mathrm{Al}$ respecto, Reddick (2005) encuentra que el nivel de ingreso de la ciudadanía impacta de forma positiva sobre la adopción del gobierno electrónico ya sea para la búsqueda de información como para el desarrollo de las transacciones más sofisticadas. En esta línea, el Banco Mundial (2016) describe que el uso del gobierno electrónico es muy desigual, los ciudadanos con el 20\% más alto de ingreso en el país de la UE más conectado son 45 veces más propensos a usar los servicios públicos electrónicos que aquellos en el $20 \%$ más bajo de ingreso correspondiente al país menos conectado de la región.

Por otro lado, Norris y Moon (2005) encontraron que en el caso de los municipios norteamericanos, el tamaño medido por la cantidad de habitantes o población está estrechamente relacionado con la implementación de los servicios online. Varios autores (Cerrano-Cinca et al. 2009, Wilkinson y Cappel 2005, Moon 2002) indicaron que cuanto más grande es el municipio es más probable que el mismo adopte mayores prácticas de gobierno electrónico comparado a los municipios pequeños. Por otro parte, cuanto mayor es la población mayor es la probabilidad de encontrar municipios con actividades participativas (Salvador 2004, Criado 2004, Blanco y Font 2005, Brown y Schelin 2005). Autores más recientes también indican que el tamaño de la ciudad importa para promover la transparencia (Albalate del Sol 2013, Bearfield y Bowman 2016). En las ciudades grandes la competencia política estimula la transparencia, mientras que en las ciudades pequeñas los recursos gubernamentales y el profesionalismo administrativo influyen en la transparencia. En la Unión Europea, los empresarios tienen más probabilidad de usar internet para interactuar con el gobierno que los ciudadanos. Sin embargo, Borge et al. (2008), tras realizar una análisis explicativo de la participación ciudadana electrónica en los municipios de la región de Cataluña, no encuentra que el nivel de ingresos sea una variable significativa. 


\section{ESTADO DEL ARTE EN ARGENTINA}

En la actualidad, Argentina es uno de los 16 países que se encuentran adscritos a la AGA (Hoffman et al. 2013, Oszlak y Kaufman 2014). El objetivo fundamental de la AGA es promover una cultura de Gobierno Abierto con el fin de lograr el empoderamiento de los ciudadanos, generar impactos a nivel económico y social, y difundir los ideales de la apertura y la participación del Siglo XXI. Los desafíos de la AGA se sustentan en los principios de transparencia, compromiso y participación ciudadana, y tecnología e innovación. La AGA también lanzó un programa piloto de gobiernos subnacionales que tenía entre sus siete miembros correspondientes a esta región a Buenos Aires (Argentina),

En Argentina, la Ley de Acceso a la Información Pública de 2016 impulsada por la sociedad civil, promovió avances fundamentales para desarrollar mecanismos más simples y accesibles para la incorporación del concepto de transparencia activa y la realización de solicitudes de información. La ley obliga en sus artículos 18 y 19 la creación de agencias de acceso a la información pública en todos los poderes del Estado (Ejecutivo, Legislativo y Judicial) y en los ministerios públicos. Sin embargo, la coordinación institucional es primordial para lograr implementar efectivamente este derecho.

Durante 2018, el Centro de Implementación para las Políticas Públicas para la Equidad y el Crecimiento (CIPPEC), con la participación de Euro Social y la Dirección Nacional de Relaciones con la Comunidad y Participación Ciudadana como líder, inició el proceso de construcción de un sistema de indicadores (Aquilino, Arena, y López Méndez 2020).

Una forma de comparar a los países o municipios de acuerdo a su grado de participación en las políticas de gobierno abierto es a través del análisis de índices compuestos. Por medio de estos datos se estable un ranking en los que se compara la posición relativa de cada caso.

En Argentina, existen publicados algunos índices relacionados con la transparencia de nivel municipal. Los municipios constituyen una de las divisiones geográficas básicas del país, mientras que las provincias representan la división territorial de primer orden. Se consideran 23 provincias en el país, y se incluye como una unidad de análisis adicional a la Ciudad Autónoma de Buenos Aires (CABA) por constituir un caso especial (Rossati 2004). El territorio de las provincias está dividido en departamentos, y estos, a su vez, integrados por municipios y/o comunas, con la excepción de la provincia de Buenos Aires que se divide en 
municipios denominados partidos (INDEC 2008). Luego, los índices de transparencia que se han publicado en Argentina son, en primer lugar, el índice de Datos Abiertos de Ciudades de Argentina (IDACA) publicado por Open Knowledge International o Fundación Conocimiento Abierto Argentina (OKFN). Este permite mensurar el estado actual de la liberación de datos en formato abierto en los municipios de Argentina. El índice se calcula sobre 24 ciudades de Argentina y toma como referencia el Global Open Data Index. Sobre un total de 240 conjuntos de datos evaluados en 24 ciudades, solo 60 ( $25 \%$ de la totalidad) cumplen con todos los criterios para ser considerados datos abiertos, por lo que hay mucho menos de la mitad de los datasets que no cumplen con la puntuación ideal. El ranking es liderado por el municipio de Córdoba con un 95\% de apertura e inmediatamente lo siguen Ciudad de Buenos Aires (91\%), Villa María (90\%) y Bahía Blanca (88\%).

El segundo es el Índice de Transparencia Presupuestaria Provincial (ITPP) publicado por CIPPEC. En él se evalúa, a través de este índice la calidad, cantidad, nivel de desagregación y actualización de la información presupuestaria que publican los gobiernos provinciales en sitios web oficiales. CIPPEC elabora el índice analizando leyes de presupuesto, informes de ejecución de gastos, informes de recaudación impositiva, informes de deuda pública, información sobre transferencias recibidas del gobierno nacional, transferencias realizadas a municipios, la Cuenta de Inversión y el Presupuesto Ciudadano.

En tercer lugar, se encuentra el ITMA. Este índice surge a partir de la exploración de los sitios web municipales para determinar la presencia o no de información presupuestaria y de gasto público, así como la existencia de un sistema centralizado de gestión de las solicitudes de información y la publicación de las respuestas para su aprovechamiento público. Según los resultados obtenidos, los municipios más transparentes son Córdoba, CABA, Bahía Blanca y Ushuaia.

Como señalan Ciucci et al. (2019: 77):

En este sentido, el ITM introduce aspectos de transparencia activa que no han sido considerados por el resto tales como información sobre subsidios y agenda abierta del intendente, y a su vez, incluye nociones de transparencia pasiva, como el derecho de solicitar información pública por parte de los ciudadanos, que exista información publicada al respecto así como un sistema online para recibir las solicitudes de información. 
Por otra parte, en materia de difusión de las TIC a nivel municipal en Argentina, se dispone de datos sobre difusión de las TIC a nivel de los hogares publicados por el INDEC. El informe técnico de INDEC (2017) brinda la siguiente información: 1) en todos los aglomerados, el porcentaje de penetración de computadoras (medida como porcentaje de hogares con computadora) supera el 50\%; 2) Los aglomerados urbanos con una penetración más alta de la computadora (superior al 70\%), corresponden a San Luis, Santa Rosa, La Rioja, La Plata y Bahía Blanca; y 3) el aglomerado urbano con un menor nivel de acceso a computadoras es Formosa. Por otro lado, los aglomerados urbanos con un nivel más alto de acceso a Internet (medido como porcentaje de hogares con Internet superior al 79\%) son La Plata, Santiago del Estero, Rawson, Mendoza y Santa Rosa. Por su parte, Catamarca es el aglomerado con nivel más bajo de acceso a Internet.

Por último, los aglomerados con un porcentaje más alto de hogares que usan computadora (porcentajes superiores al 53\%) son San Luis, La Plata, Jujuy, Catamarca y La Rioja. Se observa por lo tanto, que las tasas de acceso son superiores a las tasas de uso. En este caso, Santa Rosa es el aglomerado con el porcentaje de uso más bajo. A su vez, respecto al indicador porcentaje de hogares que usan Internet, los aglomerados de La Plata, Mendoza, Rawson, Bahía Blanca y Corrientes alcanzan los niveles más altos. Luego, La Plata es el mejor aglomerado urbano como en términos de difusión (acceso y uso) de las TIC a nivel hogar; seguido por los municipios de La Rioja y Bahía Blanca.

\section{METODOLOGÍA}

Se utiliza como variable de transparencia municipal el ITMA que publica información sobre 25 municipios de Argentina (capitales de provincia de Argentina junto con Ciudad de Buenos Aires (CABA) y Bahía Blanca.

Además del ITMA, se utiliza información sobre indicadores de TIC a nivel de los municipios capitales de provincia. Tales datos surgen de la Encuesta Nacional sobre Acceso y Uso de Tecnologías de la Información y la Comunicación (ENTIC) e INDEC (2017). Dado que no existe información de nivel municipal, se utilizan los datos de los aglomerados urbanos a los que pertenece cada municipio, de la siguiente manera:

Los indicadores TIC empleados para el análisis de conglomerado son tanto de acceso a las TIC: 1) porcentaje de hogares con computadora; 2) porcentaje de hogares con Internet; como de uso: 3) porcentaje de individuos que usa computadora; y 4) porcentaje de individuos que usa Internet. 
Finalmente, se utilizan estos datos para realizar un análisis de conglomerado como método de agrupamiento de los municipios. Este es un procedimiento estadístico multivalente que a partir de un conjunto de datos realiza una segmentación que maximiza las similitudes dentro de los grupos y maximiza las diferencias entre grupos. En otras palabras, se busca ordenar las observaciones en grupos tales que el grado de asociación natural sea alto entre los miembros del mismo grupo y bajo entre miembros de grupos diferentes.

Dado un conjunto de individuos (de $\mathrm{N}$ elementos) caracterizados por la información de $n$ variables $X j,(j=1,2, \ldots, n)$, se les aplica un algoritmo de clasificación (conglomerado) para agrupar a los municipios de la muestra que sean tan similares entre sí como sea posible (teniendo en cuenta la información disponible), siendo los distintos grupos entre ellos tan disímiles como sea posible (Pérez López 2005).

En este trabajo se realizará un clustering de k-medias que es un método no jerárquico de reasignación. El algoritmo k-medias es la técnica más empleada de particionamiento iterativo. El método de k-medias agrupa los datos dentro de $\mathrm{k}$ grupos, donde $\mathrm{k}$ es un parámetro prefijado por el investigador. Cada grupo se caracteriza por su centroide o centro que representa una media entre los elementos del grupo. El algoritmo comienza con $\mathrm{k}$ centroides, escogidos arbitrariamente, e iterativamente ejecuta los siguientes dos pasos: 1) asigna casos a los conglomerados basándose en la distancia de los centros de los conglomerados; 2) actualiza las posiciones de los centros de los conglomerados basándose en los valores medios de los casos en cada conglomerado. Estos dos pasos se ejecutan una y otra vez hasta que los datos estén ubicados en su conglomerado óptimo.

Por último, se realiza una comparación de medias (ANOVA) con la información de los conglomerados obtenidos y datos sobre el tamańo de los municipios (medido por la cantidad de habitantes) y los recursos económicos disponibles (medido por el cociente recaudación tributaria/ ingresos corrientes). Cabe aclarar que estos datos corresponden a la provincia, ya que la información disponible a nivel municipal se encuentra solo en algunos casos publicada en la sección presupuesto de los portales web municipales. Los datos utilizados a nivel provincial provienen de Fundación Libertad (2018).

El análisis de varianza (ANOVA) de un factor sirve para comparar varios grupos en una variable cuantitativa. Se trata de una generalización de la prueba t para dos muestras independientes al caso de diseños con más de dos muestras. A la variable categórica (nominal u ordinal) que define los 
grupos que se desean comparar se la denomina independiente o factor y a la variable cuantitativa (de intervalo o razón) en la que se desean comparar los grupos se la llama dependiente. La hipótesis que se pone a prueba en el ANOVA de un factor es que las medias poblacionales (las medias de la variable dependiente en cada nivel de la variable independiente) son iguales, lo que significa que los grupos no difieren en la variable dependiente y que la variable independiente es independiente de la variable dependiente (Visauta Vinacua 2007).

\section{RESULTADOS DEL ANÁLISIS DE CONGLOMERADO}

Se observa que, en promedio, el ITMA asume un valor de 0,30 para los 25 municipios analizados (Tabla 1). Este valor refleja que tan solo el 30\% de la información sobre transparencia es publicada en los sitios web de los municipios, existiendo divergencias entre municipios.

Tras aplicar el conglomerado de k-medias, se obtienen dos clusters distintos de acuerdo al ITMA y variables TIC de los conglomerados de Argentina: porcentaje de hogares con computadora, porcentaje de hogares con acceso a Internet, porcentaje de individuos que usan computara, porcentaje de individuos que usan internet y porcentaje de individuos que usan celulares. Los estadísticos descriptivos para las variables incluidas en el análisis son (Tabla 1).

Tabla 1: Estadísticos descriptivos

\begin{tabular}{|c|c|c|c|c|c|}
\hline & $\mathrm{N}$ & Mínimo & Máximo & Media & $\begin{array}{c}\text { Desviación } \\
\text { típica }\end{array}$ \\
\hline ITMA & 25 & 0,00 & 71,00 & 26,5504 & 20,05967 \\
\hline $\begin{array}{l}\text { Porcentaje de } \\
\text { hogares con } \\
\text { computadora }\end{array}$ & 25 & 52 & 86 & 66,34 & 7,873 \\
\hline $\begin{array}{c}\text { Porcentaje de } \\
\text { hogares con internet }\end{array}$ & 25 & 48 & 89 & 71,95 & 9,786 \\
\hline $\begin{array}{c}\text { Porcentaje de } \\
\text { individuos que usan } \\
\text { computadora }\end{array}$ & 25 & 49 & 81 & 67,71 & 7,771 \\
\hline $\begin{array}{c}\text { Porcentaje de } \\
\text { individuos que usan } \\
\text { Internet }\end{array}$ & 25 & 29 & 86 & 49,36 & 14,779 \\
\hline $\begin{array}{c}\mathrm{N} \text { válido (según } \\
\text { lista) }\end{array}$ & 25 & & & & \\
\hline
\end{tabular}

Fuente: Elaboración propia. 
Según el análisis de ANOVA (Tabla 2) de las variables TIC, solo resultan significativas para explicar los conglomerados el porcentaje de hogares con computadoras y el porcentaje de individuos que usan Internet. Luego, en una segunda fase se procede a realizar el análisis de conglomerados excluyendo a las variables que no resultaron significativas (Tabla 3).

\begin{tabular}{|c|c|c|c|c|c|c|}
\hline & \multicolumn{2}{|c|}{ Conglomerado } & \multicolumn{2}{|l|}{ Error } & \multirow[t]{2}{*}{$\mathrm{F}$} & \multirow[t]{2}{*}{ Sig. } \\
\hline & $\begin{array}{c}\text { Media } \\
\text { cuadrática }\end{array}$ & $\mathrm{gl}$ & $\begin{array}{c}\text { Media } \\
\text { cuadrática }\end{array}$ & $\mathrm{gl}$ & & \\
\hline ITMA & 5790,744 & 1 & 168,114 & 23 & 34,445 & 0,000 \\
\hline $\begin{array}{c}\text { Porcentaje de } \\
\text { individuos que usan } \\
\text { Internet }\end{array}$ & 1778,631 & 1 & 150,593 & 23 & 11,811 & 0,002 \\
\hline $\begin{array}{l}\text { Porcentaje de } \\
\text { hogares con } \\
\text { computadora }\end{array}$ & 535,435 & 1 & 41,399 & 23 & 12,933 & 0,002 \\
\hline $\begin{array}{c}\text { Porcentaje de } \\
\text { hogares con Internet }\end{array}$ & 210,400 & 1 & 90,774 & 23 & 2,318 & 0,142 \\
\hline $\begin{array}{l}\text { Porcentaje de } \\
\text { individuos que usan } \\
\text { computadora }\end{array}$ & 24,376 & 1 & 61,948 & 23 & 0,393 & 0,537 \\
\hline
\end{tabular}

La Tabla 3 muestra los valores finales de los centros de los conglomerados. Los valores representan las medias de las variables en cada conglomerado final. Los centros de los conglomerados finales muestran los atributos del municipio prototipo para cada conglomerado.

Tabla 3: Información de los centros de cada conglomerado

\begin{tabular}{ccc} 
& \multicolumn{2}{c}{ Conglomerado } \\
& 1 & 2 \\
\hline ITMA & 50,96 & 17,06 \\
Porcentaje de individuos que usan Internet & 63 & 44 \\
Porcentaje de hogares con computadora & 74 & 63 \\
$\mathrm{~N}$ & 7 & 18 \\
& Fuente: Elaboración propia.
\end{tabular}

El Conglomerado 1 está formado por municipios de alta transparencia y alta difusión de las TIC, con un nivel muy superior al promedio nacional en términos de transparencia municipal (con valores bastante por encima de la media) y con niveles superiores al promedio nacional en el acceso y 
uso de TIC. Está integrado por siete municipios: Bahía Blanca, Córdoba, La Rioja, San Luis, CABA, Ushuaia, y Viedma.

El Conglomerado 2 está conformado por Municipios de baja transparencia y baja difusión de las TIC, con un bajo índice de transparencia y con un nivel por debajo de las medias obtenidas de acceso y uso de TIC. Se puede observar que la mayoría de los municipios analizados pertenecen al Conglomerado 2. Este cluster es el más numeroso, con 18 municipios.

\section{RELACIÓN CON VARIABLES SOCIOECONÓMICAS Y DEMOGRÁFICAS}

A continuación, se analiza si existen divergencias en el tamaño de los municipios (medido por cantidad de habitantes) así como en el ingreso o recursos de los municipios (aproximado por los recursos de la provincia de pertenencia) según el conglomerado de pertenencia (Tabla 4).

Tabla 4: Media de habitantes por conglomerado de pertenencia

\begin{tabular}{cccc} 
& & Recursos & Población \\
\hline \multirow{2}{*}{1} & Media & 33,4571 & 736039,5714 \\
& $\mathrm{~N}$ & 7 & 7 \\
& Deviación típica & 21,28057 & 1064136,02624 \\
2 & Media & 21,7500 & 282426,8333 \\
& $\mathrm{~N}$ & 18 & 18 \\
& Deviación típica & 10,88368 & 196216,17050 \\
& Media & 25,0280 & 409438,4000 \\
& $\mathrm{~N}$ & 25 & 25 \\
& Desv. típ. & 15,03007 & 594624,51072 \\
& & Fuente: Elaboración propia.
\end{tabular}

De acuerdo a los resultados, si bien se observa que los municipios del Conglomerado 1 tienen en promedio una mayor cantidad de habitantes, la prueba de ANOVA indica que tales diferencias son estadísticamente significativas solo al 10\%. Por otro lado, dado que el tamańo del municipio no es una variable representativa del nivel de ingresos del municipio, se estableció el mismo tipo de análisis con la variable recaudación tributaria/ ingresos corrientes. El Conglomerado 1 pareciera corresponder a provincias con mayor producto, sin embargo, tal diferencia es solo significativa estadísticamente al 10\%. Es decir, la transparencia estaría más ligada al nivel de producto riqueza de la provincia. 
Tabla 5: ANOVA

\begin{tabular}{ccccccc} 
& & Suma de cuadrados & gl & Media cuadrática & F & Sig. \\
\hline Recursos & Intergrupos & 690,768 & 1 & 690,768 & 3,358 & 0,080 \\
& Intragrupos & 4730,902 & 23 & 205,691 & & \\
& Total & 5421,670 & 24 & & & \\
Población & Intergrupos & 1037053161457,790 & 1 & 1037053161457,790 & 3.202 & 0,087 \\
& Intragrupos & 7448826248630,210 & 23 & 323862010810,009 & & \\
& Total & 8485879410088,000 & 24 & & &
\end{tabular}

Fuente: Elaboración propia.

\section{DISCUSIÓN}

El análisis de conglomerados realizado permitió encontrar dos grupos o conglomerados de municipios en Argentina en función del ITMA y de las variables TIC a nivel hogares e individual. Entre las variables TIC que resultaron significativas en la construcción de los conglomerados se hallan porcentaje de hogares con Computadora y porcentaje de individuos que usan Internet. La mayoría de los municipios analizados (72\%) pertenecen al Grupo 2, que son municipios de baja transparencia y de baja difusión de las TIC. Por otra parte, el porcentaje restante corresponde al Grupo 1 formado por los municipios de mayor transparencia y difusión de las TIC. Este resultado concuerda con Alderete (2018) según el cual las TIC son un factor mediador, y por tanto necesario, para la consecución de los objetivos del gobierno abierto.

Por otro lado, de la relación de los grupos de municipios con otras variables de control, se obtiene que el tamaño del municipio, así como el nivel de ingreso de los mismos es superior en el Grupo 1 que en Grupo 2, es decir, los municipios más transparentes y de mayor penetración de las TIC son también los de mayor tamaño (por cantidad de habitantes) y con mayores recursos económicos. Estos resultados concuerdan con estudios previos tales como Bearfield y Bowman (2016), Albalate del Sol (2013), Cruz et al. (2012) y Reddick (2005). Aunque estadísticamente, ambas variables solo resultan estadísticamente significativas al 10\% para explicar tales diferencias, siguiendo a Borge et al. (2008).

\section{CONCLUSIONES}

Los ciudadanos son hoy actores partícipes importantes en la política pública, sobre todo a nivel municipal. La transparencia, como uno de los principios del paradigma de gobierno abierto, favorece la comunicación 
entre los ciudadanos y el gobierno, así como la publicación de los datos de orden público para la toma de decisiones. En este contexto, este trabajo tiene como objetivo examinar si los municipios capitales de provincia de Argentina, junto con Ciudad de Buenos Aires y Bahía Blanca, pueden ser agrupados siguiendo algún patrón de comportamiento en torno a los niveles de transparencia y de difusión de las TIC. A partir de un análisis de conglomerados, se hallan dos grupos o conglomerados de municipios, los municipios de alto porcentaje de transparencia y de TIC, y los municipios de baja transparencia y de TIC.

Por otro lado, se examina si existe alguna diferencia estadísticamente significativa entre los grupos o conglomerados encontrados y el perfil de los municipios en relación al tamaño y a los recursos económicos disponibles. En principio, según el análisis de varianza (ANOVA), los conglomerados poseen relación con el tamaño de los municipios (medido por cantidad de habitantes) así como con el nivel de recursos disponibles. Este resultado indicaría que las políticas de modernización de la administración pública de nivel municipal pueden resultar más exitosas en algunos tipos de municipios, pero no en todos. Es probable que en el caso de municipios pequeños y con escasos recursos, las políticas de transparencia y de difusión de las TIC sean más difíciles de implementar.

Entre las limitaciones del trabajo, se encuentran que el trabajo mide transparencia (tanto activa como pasiva) utilizando un solo índice (el ITMA). Sería útil contrastar los resultados con índices alternativos para dar robustez a las relaciones halladas. Por otro lado, este trabajo solo realiza un análisis exploratorio; no es posible plantear relaciones causales entre las variables estudiadas. Para tal fin, sería necesario un mayor número de observaciones, ya que el número de capitales de provincia es reducido.

La principal contribución de este trabajo consiste en analizar el perfil de los municipios en torno a las políticas de transparencia. Mediante esta tipificación, los municipios pueden evaluar su posición relativa en materia de transparencia. De esta manera, se puede promover la realización de diagnósticos de las políticas locales para comprender las acciones requeridas para alcanzar la transparencia. 


\section{REFERENCIAS}

Ackerman, J. M. y Sandoval-Ballesteros, I. E. (2006). The global explosion of freedom of information acts. Administrative Law Review, 58 (1), 85-130.

Albalate del Sol, D. (2013). The institutional, economic and social determinants of local government transparency. Journal of Economic Policy Reform, 16, 90-107.

Albino. V., Berardi. U., y Dangelico, R. M. (2015). Smart cities: Definitions. dimensions. Performance and initiatives. Journal of Urban Technology. 22, 3-21.

Alderete, M. V. (2018). The mediating role of ICT in the development of open government Journal of Global Information and Technology Management, 21 (3), 172-187.

Alderete, M. V. y Linares, S. (2017). Participación ciudadana: el caso de la Ordenanza de Acceso a la Información Pública de la Municipalidad de Bahía Blanca. Ponencia presentada en el LII Reunión Anual de la Asociación Argentina de Economía Política, 15-17 de noviembre, Bariloche, Argentina.

Anthopoulos, L. y Fitsilis, P. (2010). From Digital to Ubiquitous Cities: Defining a Common Architecture for Urban Development. Proceedings of the 6th International Conference on Intelligent Environments IE'10, Malaysia 2010, IEEE.

Aquilino, N., Arena, E. y López Méndez, E. (2020). Programa de monitoreo y evaluación. Medir el acceso a la información línea de base de la política nacional de acceso a la información. Buenos Aires: CIPPEC.

Aström, J., Karlsson, M., Linde, J. y Pirannejad, A. (2012). Understanding the rise of e-participation in non-democracies: domestic and international factors. Government Information Quarterly, 29 (2), 142150.

Banco Mundial (2016). Digital dividens. Overview. World development report 2016. Nueva York: Banco Mundial. 
Bearfield, D. A. y Bowman, A. (2017). Can you find it on the web? An assessment of municipal e-government transparency. American Review of Public Administration, 47 (2), 172-188.

Blanco, I. y Font, J. (2005). ¿Qué hay detrás de la oferta de participación? El rol de los factores instrumentales e ideológicos en los mecanismos españoles de participación. Revista del CLAD Reforma y Democracia, $31,1-17$.

Borge, R.; Colombo, C. y Welp, Y. (2008). Análisis explicativo de la participación ciudadana electrónica y presencial en el ámbito municipal de Cataluña IDP. Revista de Internet, Derecho y Politica, 6, 13-31.

Brewer, G.A., Neubauer, B.J. y Geiselhart, K. (2006). Designing and implementing e-government systems. Critical implications for public administration and democracy. Administration \& Society, 38 (4), 472479 .

Brown, M. M. y Schelin, S. (2005). American local governments: confronting the e-government challenge. En Drüke, H. (Ed.), Local electronic government: A comparative study. Nueva York: Routledge.

Camisón-Zornoza, C., Lapiedra-Alcamí, R., Segarra-Ciprés, M.M. y Boronat-Navarro, M. (2004). A meta-analysis of innovation and organizational size. Organization Studies, 25 (3), 331-336.

Ciucci, F., Díaz, L, Alderete, M.V. y Linares, S. (2019). Construcción de un índice para medir la transparencia municipal: Buenos Aires, Bahía Blanca y las capitales de provincia de Argentina. Revista Iberoamericana de Estudios Municipales, 20, 59-84.

Centro Latinoamericano de Administración para el Desarrollo (CLAD). (2016). Carta Iberoamericana de Gobierno Abierto, aprobada por la XVII Conferencia Iberoamericana de Ministras y Ministros de Administración Pública y Reforma del Estado. Disponible en https://www.clad.org/ images/declaraciones/CIGA-Octubre-2016.pdf [01-12-2020].

Cerrillo i Martínez, A. (2005). La Gobernanza hoy: 10 textos de referencia. Madrid: INAP.

. (2012). La contribución de las TIC a la mejora de la transparencia administrativa. Arbor Ciencia, Pensamiento y Cultura, 188 (756), 707-724. 
Chen, C.W. (2010). Impact of quality antecedents on taxpayer satisfaction with online tax- filing systems-An empirical study. Information \& Management, 47, 308315.

Concha, G. y Naser, A. (2012). Datos abiertos: un nuevo desafío para los gobiernos de la región. CEPAL. Serie Gestión Pública N74. Santiago: CEPAL.

Criado, J. I. (2004). Construyendo la e-Administración local. Madrid, EuroGestión Pública.

. (2016). Las administraciones públicas en la era del gobierno abierto. Gobernanza inteligente para un cambio de paradigma en la gestión pública. Revista de Estudios Políticos, 173, 245-275.

Cullier, D. y Piotrowski, S. J. (2009). Internet information-seeking and its relation to support for access to government records. Government Information Quarterly, 26 (3), 441-449.

Curtin, D. y Meijer, A. J. (2006). Does transparency strenghen legitimacy? Information Polity, 11 (2), 109-122.

Curtin G. G., Sommer JD, M. H. y Vis-Sommer, V. (2003). The world of e-government. Journal of Political Marketing, 2 (3-4), 1-16.

Cruz, C. F., Ferreira, A. C., de S., Silva, L. M. da, y Macedo, M. Álvaro da S. (2012). Transparência da gestão pública municipal: um estudo a partir dos portais eletrônicos dos maiores municípios brasileiros. Revista de Administração Pública, 46 (1), 153-176.

Davis, F. (1989). Perceived Usefulness, perceived ease of use and user acceptance of information technology. MIS Quarterly, 13 (3), 319340.

Dewan S. y Riggins F. J. (2005). The digital divide: Current and future research directions Journal of the Association of Information Systems, 6 (12), 298-337.

Ebbers, W., Jansen, M.G.M. y van Deursen, A. (2016). Impact of the digital divide on e-government: Expanding from channel choice to channel usage. Government Information Quarterly, 33 (4), 685-692. 
Farioli, M. I. (2015). La transparencia y el derecho de acceso a la información pública en Argentina. Tesis de Magister (Administración Pública), Universidad Nacional del Litoral, Argentina.

Fenster, M. (2010). Seeing the State: Transparency as metaphor. Administrative Law Review, 62 (3), 617-628.

Fundación Libertad (2018). Indice de desempeño provincial 2018. Buenos Aires: Fundación Libertad.

Fung, A., Graham, M. y Weil, D. (2007). Full disclosure. The perils and promise of transparency. Nueva York: Cambridge University Press.

García-Sánchez, I.M., Rodríguez-Domínguez, L. y Frías Aceituno, J.V. (2013). Evolutions in e-governance: Evidence from Spanish local governments. Environmental Policy and Governance, 23, 323-340.

Grant, G. y Chau, D. (2005). Developing a Generic Framework for E-Government. Journal of Global Information Management, 13 (1), $1-30$.

Grimmelikhuijsen, S. (2009). Do transparent government agencies strengthen trust? Information Polity, 14 (3), 173-186.

Grönlund, A. y Horan, T. (2004).Introducing e-gov: History, definitions, and issues. Communications of the Association of Information Systems, $15,713-729$.

Gupta, K.P., Singh, S. y Bhaskar, P. (2016). Citizen adoption of e-government: a literature review and conceptual framework. Electronic Government, An International Journal, 12 (2), 160-185.

Helbig, N., Gil-García, J. R. y Ferro, E. (2009). Understanding the complexity of electronic government: Implications from the digital divide literature. Government Information Quarterly, 26 (1), 89-97.

Herrero, A. y Gentili, R. (2015). Acercando el gobierno abierto al ciudadano: herramientas de gobierno abierto para Estados provinciales y municipales. Disponible en: http://conie.chaco.gov.ar/publicaciones_electronicas/ trabajos/3.30.pdf [01-12-2020].

Hernández Bonivento, J. (2016). Diseño institucional para el gobierno abierto municipal: propuesta de medición y análisis del caso chileno. 
Documentos y Aportes en Administración Pública y Gestión Estatal, 16 (27), 101-128.

Hofmann, A., Ramírez Alujas, Á. y Bojórquez, J. (2013). La promesa del Gobierno Abierto. México: ITAIP.

Instituto Nacional de Estadística y Censos (INDEC) (2008). Superficie y cantidad de departamentos, por provincia (xls). Territorio/geografía. Disponible en http://www.indec.gob.ar/ [01-12-2020].

(2017). Informe Técnico del INDEC Acceso y uso de tecnologías de la información y la comunicación. EPH 2017. Disponible en http://www.indec.gob.ar/indec/web/Nivel3-Tema-4-26 [01-12-2020].

Jaeger, P. T. (2003). The endless wire: e-government as global phenomenon. Government Information Quarterly, 20 (4), 323-331.

Ke, W., y Wei, K.K. (2004). Successful e-government in Singapore. Communications of the ACM, 47 (6), 95-99.

Khalil, O. E. (2011). E-government readiness: does national culture matter? Government Information Quarterly, 28 (1), 388-399.

Kim, P. S., Halligan, J., Cho, N., Oh, C. H. y Eikenberry, A.M. (2005). Toward participatory and transparent governance: Report on the Sixth Global Forum on Reinventing Government. Public Administration Review, 65 (6), 646-654.

Kolstad, I.y Wiig, A. (2009). Is transparency the key to reducing corruption in resource-rich countries? World Development, 37, 521-532.

Lau, T. Y., Aboulhosonb, M., Linc, C., y Atkinc,D. J. (2008). Adoption of e-government in three Latin American countries: Argentina, Brazil and Mexico. Telecommunications Policy 32 (2008) 88-100.

Mariñez, F. (2017). Innovación pública en América Latina: Conceptos, experiencias exitosas, desafíos y obstáculos. Revista de Gestión Pública, $6(1), 5-18$.

Meijer, A. (2009). Understanding modern transparency. International Review of Administrative Sciences, 75 (2), 255-269 
knowledge society. Nueva York: Springer

. (2012). eDemocracy \& e Government. Stages of a democratic

Montoya Morales, L.M. y Zhardez, V. (2016). Gobierno Local: construcción colectiva del conocimiento para la modernización municipal. Documento para su presentación en el VII Congreso Internacional en Gobierno, Administración y Políticas Públicas GIGAPP, 3-5 de octubre, Madrid, España.

Moon, M. J. (2002). The evolution of e-government among municipalities: rhetoric or reality? Public Administration Review, 62 (4), 424-433.

Naser, A. y Ramirez-Alujas, A. (2017). Plan de gobierno abierto. Una hoja de ruta para los Gobiernos de la región. Santiago: CEPAL.

Norris, D. y Moon, J. (2005). Advancing e-government at the grassroots: Tortoise or hare? Public Administration Review, 65 (1), 64-74.

Noveck, B. S. (2009). Wiki government: How technology can make government better, democracy stronger, and citizens more powerful. Harrisonburg, VA: Brookings Institution Press.

Oszlak, O. y Kaufman, E. (2014). Teoría y práctica del gobierno abierto: Lecciones de la experiencia internacional. OEA, 2015. Disponible en: http://redinpae.org/recursos/kaufmanoszlak.Pdf [01-12-2020].

Pérez López, C. (2005). Métodos estadísticos avanzados con SPSS. Madrid: Thompson.

Rogers, E. M. (1995). Diffusion of innovations. Nueva York: Free Press.

Ramírez-Alujas, Á. y Güemes, M. C. (2013). Gobierno abierto: oportunidades y desafíos. Una reflexión socio-política con la mirada puesta en Latinoamérica. Ponencia presentada en el XV Congreso Internacional América Latina: La autonomía de una región, 29-30 de noviembre, Madrid, España

Reddick, C. (2005). Citizen interaction with e-government: From the streets to servers? Government Information Quarterly, 22, 38-57.

Salvador, M. (2004). Midiendo el gobierno electrónico: un análisis de las páginas web de las corporaciones locales desde la perspectiva del ciudadano. Ponencia presentada en el IX Congreso del CLAD sobre 
reforma del Estado y de la administración pública, 2-5 noviembre, Madrid, Espańa.

Siau, K. y Long, Y. (2009). Factors impacting e-government development. Journal of Computer Information Systems, 50 (1), 98-107.

Singh, H., Das, A. y Damien, J. (2007). Country level determinants of e-government maturity. Communications of the Association for Information Systems, 20 (1), 632-648.

Serrano-Cinca, C., Rueda-Tomás, M. y Portillo-Tarragona, P. (2009). Determinants of e-government extension. Online Information Review, 33 (3), 476-498.

Teo, T. S., Srivastava, S. C. y Jiang, L. (2008). Trust and electronic government success: An empirical study. Journal of Management Information Systems, 25 (3), 99-132.

Van Deursen, A. y Van Dijk, J. (2011). Internet skills and the digital divide. New Media \& Society, 13 (6), 893-911.

Villoria, M. y Ramírez Alujas, Á. (2013). Los modelos de gobierno electrónico y sus fases de desarrollo: Un análisis desde la teoría política. Gestión y Política Pública, 22 (número especial), 69-103.

Visauta Vinacua, B. (2007). Análisis estadístico con SPSS 14: Estadistica básica. Madrid: McGraw-Hilll Interamericana.

Weerakkody, V., Irani, Z., Lee, H., Hindi, N. y Osman, I. (2016). Are U.K. citizens satisfied with e-government services? Identifying and testing antecedents of satisfaction. Information Systems Management, 33 (4), 331-343.

Wilkinson, V. O. y Cappel, J. J. (2005). Impact of Economic Prosperity and Population on EGovernment Involvement. Information Systems, 6 (2), 204-209.

Recibido: 16-07-2020

Aceptación de la versión final: 01-12-2020 